\title{
Atomistic Mechanisms of Morphological Evolution and Segregation in U-Zr Alloys
}

\author{
A. P. Moore ${ }^{\mathrm{a}, *}$, C. Deo ${ }^{\mathrm{a}}$, M. I. Baskes ${ }^{\mathrm{b}, \mathrm{c}, \mathrm{d}}$, M. A. Okuniewski ${ }^{\mathrm{e}} \mathrm{f}$ \\ ${ }^{a}$ Georgia Institute of Technology, 770 State Street, Atlanta, GA 30332, USA \\ ${ }^{b}$ Mississippi State University, Mississippi State, MS 39762, USA \\ ${ }^{c}$ University of California, San Diego, 9500 Gilman Drive, La Jolla, CA 92093, USA \\ ${ }^{d}$ Los Alamos National Laboratory, PO Box 1663, Los Alamos, NM 87545, USA \\ ${ }^{e}$ Purdue University, 610 Purdue Mall, West Lafayette, IN 47907 \\ ${ }^{f}$ Idaho National Laboratory, PO Box 1625, Idaho Falls, ID 83415, USA
}

\begin{abstract}
In many metallic alloys, complex microstructures form as a consequence of local atomic ordering that depends on the processing path. This research uses an atomistic approach to study microstructural morphology and evolution in order to investigate how temperature and alloy concentration affect ordering. A semi-empirical Modified Embedded Atom Method (MEAM) is used in conjunction with molecular dynamics (MD) and Monte Carlo (MC) simulations to investigate the properties and equilibrium configurations of the high temperature body-centered-cubic (bcc) uranium-zirconium (U-Zr) alloys. Atomic simulations conducted with the MEAM potential show the thermodynamic driving force to the lamellar structure for the melt-casted U-rich alloys and the finely acicular microstructure of the water quenched U-rich alloys. In addition, when the U-rich U-Zr alloy is equilibrated at a lower temperature, the lamellar/acicular microstructures begin to spheroidize, as observed in experiments. In the intermediate Zr concentration region, the ordering seen is able to facilitate the structure to the partially ordered $\delta$ - $\mathrm{UZr}_{2}$ phase seen experimentally. Lastly, the Zr-rich region is able to successfully show the thermodynamic driving force to the acicular, Widmanstätten, and martensitic needles type microstructures observed experimentally. These simulations are able to successfully replicate some of the fundamental thermo-physical and microstructural characteristics following fabrication and irradiation of the U-Zr metallic fuels.
\end{abstract}

Keywords: Microstructure Evolution, Metal Alloys, Uranium, Zirconium, Atomistic Simulations

\section{Introduction}

Microstructural evolution in metallic alloys involves complex atomic processes occurring over a wide range of temporal and spatial scales. Underlying the evolution of microstructure are unit mechanisms involving atoms or groups or atoms that diffuse in order to satisfy local thermodynamic constraints. Complex microstructures form as a consequence of local atomic ordering that depends on the processing path.

\footnotetext{
${ }^{*}$ Corresponding author.

Email address: amoore31@gatech.edu (A. P. Moore)
}

(C) 2016. This manuscript version is made available under the Elsevier user license http://www.elsevier.com/open-access/userlicense/1.0/ 
An example of such complex microstructure formation is the uranium-zirconium (U-Zr) system, where ordering and phase separation effects are observed and cause microstructures to be highly dependent on processing paths. Fig. 1 shows the binary U-Zr phase diagram with important major phase regions indicated. A U-Zr microstructural characteristic is the uranium separation or decomposition from the U-rich $\mathrm{U}-\mathrm{Zr}$ alloy to form the $\alpha$-U and $\delta$ - $\mathrm{UZr}_{2}$ phases in various formations. Five representative microstructures are schematically shown in conjunction with the phase diagram.

For example, if the U-rich alloy is air-cooled from the melt, it results in a lamellar microstructure. The same alloy, quenched from the high temperature bcc phase, forms a more acicular structure while an alloy with a slightly higher Zr concentration, quenched from the miscibility gap, results in spherical decomposition. The lamellar microstructure of U-rich U-Zr alloys has been noticed by Basak et al., McKeown et al. and Kim et al. [1 3]. In each case, this lamellar microstructure formed when the alloy was heated above its melting temperature and then cooled at a modest rate, not quenched [1-3]. However, if the sample was very rapidly quenched, there is evidence that the bcc $\gamma$-U phase may hold instead of decomposing [4], and if cooled quickly may either decompose into $\alpha$-U and $\delta$-UZr 2 phases or form a supersaturated $\alpha^{\prime}$-phase in a Widmanstätten morphology with fine interleaving lamellae [5, 6].

The Zr-rich U-Zr alloys also decompose. Previous publications [7-10] have classified some of the resulting microstructures of this decomposition as acicular, Widmanstätten, disc, globular, rhomboidal and more complex decompositions. If water-quenched from the bcc solid solution region, the Zr-rich U-Zr can remain in the bcc phase [7, 8, with possible martensitic $\alpha$-Zr needles or bainitic $\alpha$-Zr precipitates [7. However, if air-cooled, Zr-rich alloys result in an acicular decomposition, and if furnace-cooled, in either a Widmanstätten or rhomboidal microstructure after decomposition of the $\alpha$-Zr [7, 8]. If the sample is annealed in the $\alpha$-Zr, $\gamma$-U-Zr temperature region, either a globular or rhomboidal, or both, $\alpha$-Zr forms in the $\delta$ - $\mathrm{UZr}_{2}$ phase [7-10]. 

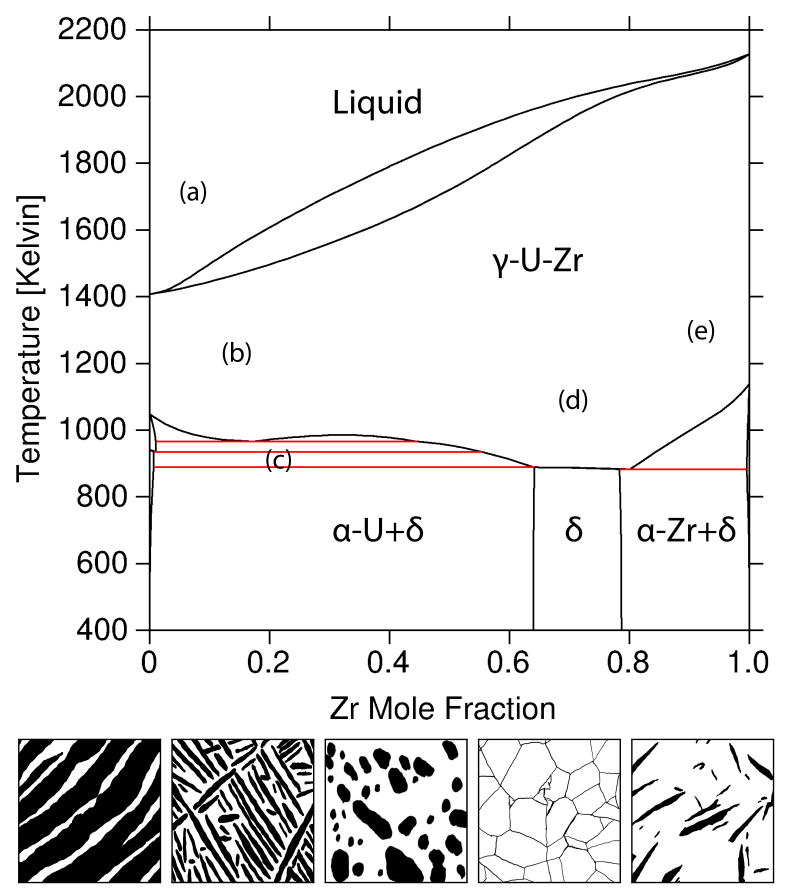

(a)

(b)

(c)

(d)

(e)

Figure 1: Binary U-Zr phase diagram, with important major phase regions indicated and representations of observed microstructures if cooled with a given rate from a temperature range. The phase diagram was produced using Thermo-Calc and the TCBin database [11] 12, which uses the previously published thermodynamics and phase diagrams [13] 15].

(a): (U-rich) melt to low air-cooled: results in lamellar

(b): (U-rich) bcc quenched: results in acicular

(c): (U-rich) $\alpha$-U, $\gamma$-U-Zr phase region quenched: results in spherical decomposition

(d): (Intermediate) bcc quenched: results in equiaxed

(e): (Zr-rich) bcc quenched: results in bcc with martensitic needles

Evidently, atomistic configurations in the bcc and liquid phases, where atomic diffusion is significant, is key in the research of microstructure and morphological evolution of the U-Zr system. Neither U, $\mathrm{Zr}$, nor their alloys lend themselves easily to atomistic analysis. Uranium is an actinide that has three distinct stable solid phases. The transition between these phases can be attributed to the behavior of the de-localized $\mathrm{f}$ orbital-electrons. The ground state uranium phase is the $\alpha$ (orthorhombic) phase. As temperature increases, uranium will transition to the $\beta$ (tetragonal) and the $\gamma$ (body-centered-cubic) phases respectively. The transition temperatures of uranium from $\alpha$ to $\beta, \beta$ to $\gamma$, and $\gamma$ to liquid are $940.85 \mathrm{~K} \pm 1.3 \mathrm{~K}, 1047.95 \mathrm{~K} \pm 1.6 \mathrm{~K}$, and $1405.95 \mathrm{~K} \pm 0.8 \mathrm{~K}$ respectively [16]. Zirconium is also a transition metal with two distinct solid phases: the ground state $\alpha$ hexagonally closed packed (hcp) phase, and the $\beta$ high temperature body centered cubic (bcc) phase (above 1139K). The U-Zr alloy transitions from a $\delta$ (partially ordered C32 crystal structure) to $\gamma$ (bcc) phase around $65 \%-75 \% \mathrm{Zr}$ and $890 \mathrm{~K}$. The $\delta-\mathrm{UZr}_{2}$ phase is a modified $\mathrm{C} 32\left(\mathrm{AlB}_{2}\right)$-type crystal structure, and is the only stable intermetallic phase in the 
U-Zr system. This C32 intermetallic crystal structure has been found in a few uranium metallic alloys, such as $\mathrm{UZr}_{2}, \mathrm{UHg}_{2}, \mathrm{U}_{3} \mathrm{Si}_{5}, \mathrm{U}_{2} \mathrm{Ti}_{0.82} \mathrm{Zr}_{0.18}, \mathrm{U}-\mathrm{Hf}$, and $\mathrm{U}_{2} \mathrm{Ti}$ [17 25]. The $\delta$ - $\mathrm{UZr}_{2}$ phase was proposed to be a modified partially ordered C32 phase by Silcock [26] and Boyko [23] using x-ray diffraction analysis.

Using atomistic simulations we attempt to understand these microstructural effects through analyzing small configurations of U-Zr alloys and looking for thermodynamic driving forces and unit mechanisms of ordering and phase separation. U-Zr interactions are described by a recently developed interatomic potential that is capable of computing several thermodynamic properties of the $\mathrm{U}$, $\mathrm{Zr}$, and $\mathrm{U}-\mathrm{Zr}$ metals and alloys [27.

The first published density functional theory (DFT) simulation of the U-Zr system was performed by Landa et al. 28] who employed the Korringa-Kohn-Rostoker method within the atomic-sphere approximation (KKR-ASA) combined with the coherent potential approximation (CPA) in order to investigate the compositional disorder. Effective cluster interactions that were used in the Monte Carlo simulations were obtained using the screened generalized perturbation method (SGPM). The first and second nearest neighbor (hereafter referred to as $1 \mathrm{NN}, 2 \mathrm{NN}, \ldots$ ) effective pair interactions (EPI) obtained by Landa et al. 28] showed significant negative values indicating a strong tendency of phase separation of the $\gamma$-U-Zr system at $0 \mathrm{~K}$.

In 2010, Bozzolo et al. 29] constructed a Bozzolo-Ferrante-Smith (BFS) potential that utilizes the equivalent-crystal theory (ECT) to examine the heat of formation, cohesive energies, and lattice parameters. This is done by utilizing the pure element cohesive energy, the bulk modulus, and the equilibrium lattice parameter, as well as the strain energy, the chemical energy for the compositions, and a reference chemical energy to obtain the formation energy of the system. The parameters needed for the BFS potential were obtained using the Linearized Augmented Plane Wave method (LAPW). The results obtained for 0K were similar to that obtained by Landa et al. 28]. Then in 2011 and 2012, the BFS potential was used to investigate lanthanide migration and possible cladding interactions [30, 31.

In 2013 Xie et al. [32] and Xiong et al. [33] called into question the enthalpy of mixing obtained by Landa et al. and Bozzolo et al. by showing that the spin orbit-coupling (SOC) and the introduction of the $\mathrm{DFT}+\mathrm{U}$ significantly changed the enthalpy of mixing and produced better matches to the experimental energetics and thermodynamics.

In 2013, Moore et al. 34] introduced a preliminary bcc U-Zr modified embedded atom method (MEAM) potential; however, it was unable to capture much of the experimental high temperature thermodynamics, and in 2015, Moore et al. 27] published a U-Zr MEAM potential designed to mimic experimental thermodynamics. It successfully replicated the enthalpy of mixing obtained by Chiotti et al. [35], as well as the elastic constants, defect formation energies, lattice constants, thermal expansion values, melting point temperatures, and enthalpy of fusion.

In what follows, we briefly describe the interatomic potential and the simulation methodology (section 
22. Using molecular dynamics and Monte Carlo simulations, independently as well as in conjunction, we analyze separation and precipitate morphology obtained through our simulations (section 3). Ordering analysis is conducted by using a short range ordering parameter, radial distribution functions, and bond order distribution functions, as well as some visual analysis. First, a comparison of (pure) $\gamma$-U and liquid-U structures is conducted to previously published Quantum Molecular Dynamics results (section 3.1). Then ordering, separation, and precipitate morphology in U-Zr alloys are studied (sections $3.2,3.3$ and 3.4. Wherever possible we compare our results with experimental or simulation results.

\section{Computational Procedure}

Atomistic simulations are performed using molecular dynamics and Monte Carlo simulations combined with the Zr MEAM potential developed by Moore et al. [27. Molecular dynamics results in step by step time snapshots of the atomic locations using the classical equations of motion, where the force on each atom is calculated using the interatomic semi-empirical MEAM potential.

The MD simulations were performed in an isothermal-isobaric (NPT) ensemble where the number of atoms, pressure, and temperature were held constant, using a 2 fs time step for 100 ps with all properties averaged over the last 35 ps. A Nose-Hoover thermostat [36, 37] was applied in the MD simulation to keep the system at a constant temperature.

In order to investigate the random to order/separated phenomena, a Metropolis MC [38, 39] simulation is performed. The MC code allows for $\mathrm{U}$ and $\mathrm{Zr}$ atoms to be interchanged in the $\mathrm{U}$-Zr system in an attempt to find the low energy configurations at a specified finite temperature. The MC code is employed in a canonical or NVT ensemble where the number of atoms, volume, and temperature is conserved. A finite series of configuration transformations (or steps) is used to drive the system to the thermodynamically equilibrated low energy states. In each MC step, one of the following two configuration changes is attempted with an equal probability:

1. A randomly selected atom is displaced from its original position in a random direction with a distance between 0 and $\mathrm{r}_{\max }$. This step accounts for the positional relaxation process (adjustment of bond lengths and angles). $\mathrm{r}_{\max }$ was chosen to be $0.35 \AA$.

2. Two randomly selected atoms with different elemental types are exchanged. This step accounts for the compositional relaxation process.

In order to determine if the configurational change to the system is accepted, the energy between the new and old configurations is evaluated. If the new configuration has a lower energy, the configurational change is always accepted. However, if the new configuration has a higher energy than the old 
configuration, the configurational change is accepted with a probability $\mathrm{P}_{X Y}$.

$$
P_{X Y}=\exp \left(\frac{-\Delta E}{k_{B} T}\right)
$$

Where $\mathrm{k}_{B}$ is the Boltzmann's constant, $\Delta \mathrm{E}$ is the change in energy between the new and old configurational states, and $\mathrm{T}$ is the temperature. Initially, during the approach to the low energy equilibrated states, the potential energy and the configurational acceptance rate will rapidly decrease following an exponential decay progression. Once the MC simulation has reached the condition of oscillating around the low energy equilibrated states, the probability of configurational transformation acceptance remains stable proportionately to the Boltzmann's distribution of the equilibrium ensemble. The $0 \mathrm{~K} \mathrm{MC} \mathrm{simu-}$ lations are performed with no Boltzmann's distribution of accepting positive energy changes; therefore, only changes that result in a decrease in the energy of the system are accepted.

The runs were conducted on a bcc 10x10x10 periodic supercell for a total of 2000 atoms. The simulation size chosen allowed for the precipitation morphology to develop in a recognizable shape, while keeping computational time reasonable. In order to test the simulation size effect, the MD and MC simulations were performed on bcc $7 \times 7 \times 7,10 \times 10 \times 10$, and $15 \times 15 \times 15$ supercells of U-20at.\%Zr at 1100K. The three different sizes lead to the same energy per atom and separation morphology, the only difference being morphological dimensions.

\section{Results and Discussion}

\section{1. bcc and Liquid $U$}

In order to develop confidence in our methods, we start by simulating pure $\mathrm{U}$ in the $\gamma$-U and liquid-U phases and compare our results with EAM and QMD results [40, 41. Elemental U is equilibrated at various temperatures in these configurations. The resulting structure is analyzed using the radial distribution function (RDF) $\mathrm{g}(\mathrm{r})$ and bond-angle distribution function $(\mathrm{BADF}) \mathrm{g}_{3}(\theta)$. The radial distribution function $\mathrm{g}(\mathrm{r})$ is defined as the relative probability of finding an atom at a distance $\mathrm{r}$ from another atom, and the bond angle distribution function $g_{3}(\theta)$ is defined as the relative probability of finding a bond angle $\theta$ an atom makes with its neighbors located within a maximum bond length [42. The $\mathrm{g}(\mathrm{r})$ and the $g_{3}(\theta)$ are calculated at various temperatures for the pure elemental liquid and bcc $\mathrm{U}$. The $\mathrm{g}_{3}(\theta)$ was calculated using a $4.2 \AA$ maximum bond length $\left(\mathrm{R}_{m}\right)$, which was determined by approximating the first minimum in $\mathrm{g}(\mathrm{r})$ (Fig. 2). The effect the maximum bond length has on the BADF can be seen in Fig. 3

Fig. 3 showing the BADF as a function of radial distance allows for discrimination of $1 \mathrm{NN}$ and $2 \mathrm{NN}$. The 55 degree peak, which is the largest peak, is due to the angle between the $1 \mathrm{NN}$ and $2 \mathrm{NN}$ atoms. Then there are the 70 and 109 degree peaks, which are due to the angle between the $1 \mathrm{NN}$ atoms. The 90 and 173 degree peaks correspond to the angle between the 2NN atoms. Lastly, the 122 degree peak corresponds to the angle between the $1 \mathrm{NN}$ and $2 \mathrm{NN}$ atoms. 
The melting transition to the liquid phase from the solid bcc phase is not forced, but is allowed to occur naturally. The transition to the liquid state can be determined using the $\mathrm{g}_{3}(\theta)$ (Fig. 4) as seen in the $1400 \mathrm{~K}$ and $2150 \mathrm{~K}$ for pure $\mathrm{U}$. The bonding angles from the solid bcc phase to the liquid phase change significantly. In the liquid phases, the bonds change to a more bimodal distribution with peaks around $55^{\circ}$ and $110^{\circ}$ ending with a trailing tail.

The $\mathrm{g}(\mathrm{r})$ (Fig. 2) and the $\mathrm{g}_{3}(\theta)$ (Fig. 4) structural data obtained from the MD MEAM potential's runs for $\mathrm{U}$ is compared against those obtained using a quantum molecular dynamics (QMD) method by Hood et al. [40] and to the embedded atom method (EAM) by Smirnova et al. [41. The MEAM and QMD g(r) both change smoothly with increasing temperature, indicating no solid-solid transitions. Similar to these studies, we find that the last peak in the $g_{3}(\theta)$ around $180^{\circ}$, characteristic of a cubic or tetragonal solid, disappears from the bcc solid to the liquid state for U. In addition, the liquid states also show a bond-angle short range ordering (SRO), resulting in a characterizable structure (see Fig. 5). The liquid structure is found to be a collection of tetrahedra with common vertices, whose bond-angle SRO decreases slowly as the temperature of the liquid increases.

The MEAM shows similar trends to the $\mathrm{g}(\mathrm{r})$ and the $\mathrm{g}_{3}(\theta)$ of the QMD and EAM results. There are however a few discrepancies that can be attributed to the MEAM having a lower melting point than the EAM and the experimental melting point of 1407.95K [16]. We estimate that the MEAM melting point is between 1325-1350K [27, while the EAM has a melting point around 1500K [41. The QMD melting point was not given [40].

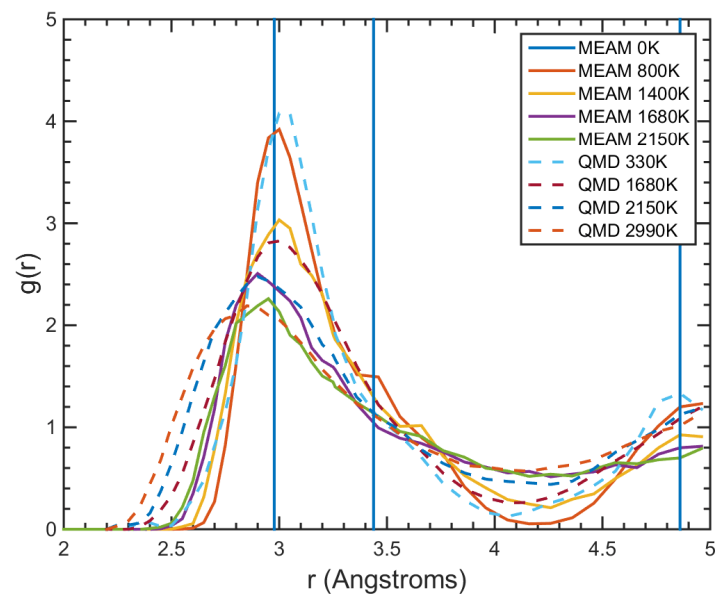

Figure 2: RDF g(r) for uranium calculated at different temperatures using the MEAM potential in MD simulations compared with the QMD results. The vertical lines are the RDF positions for the MEAM potential at OK. 


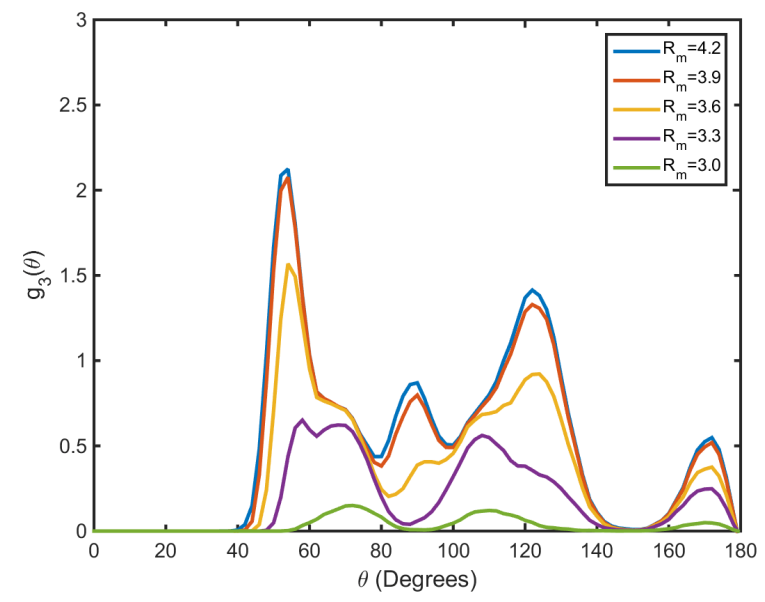

Figure 3: $\mathrm{BADF}_{3}\left(\theta, \mathrm{R}_{m}\right)$ for the bcc $\mathrm{U}$ phase at $800 \mathrm{~K}$, calculated for different values of the maximum bond length $\mathrm{R}_{m}$.

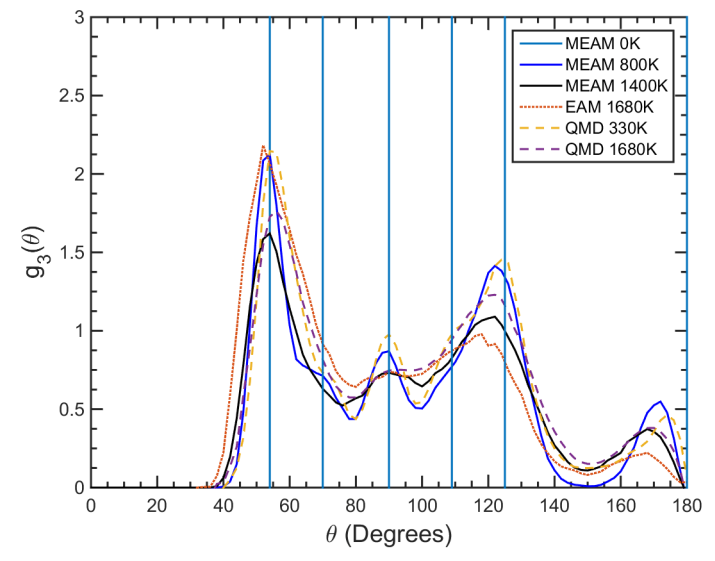

(a)

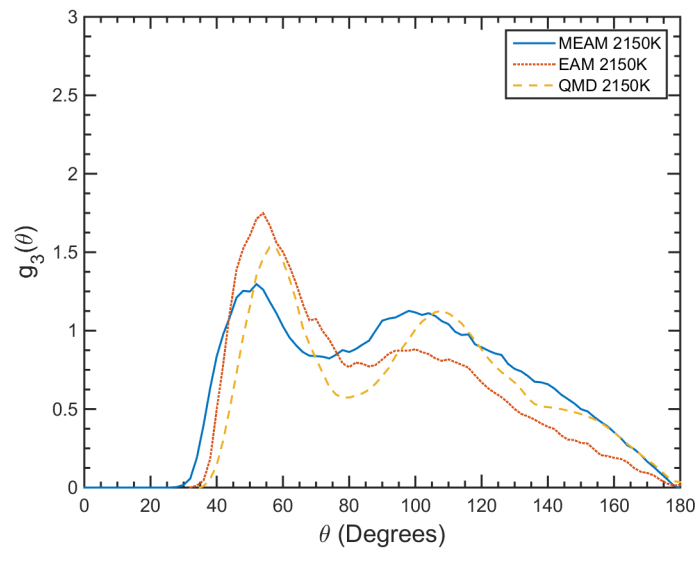

(b)

Figure 4: $\mathrm{BADF}_{3}(\theta)$ for (a) bcc uranium calculated at different temperatures and for (b) liquid uranium calculated at 2150K using the EAM, MEAM, and QMD results. The vertical lines are the BADF positions for the MEAM potential at $0 \mathrm{~K}$.

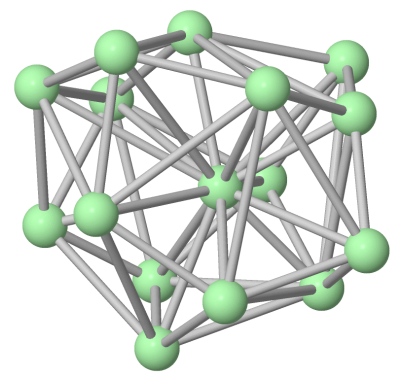

Figure 5: Snapshot of the liquid-U structure at $2300 \mathrm{~K}$, showing the bonds within a $4.2 \AA$ maximum bond length $\left(\mathrm{R}_{m}\right)$ of the central atom and the network of tetrahedrals characterizing the bonding in the liquid phases. 


\subsection{Ordered/Disordered $U-Z r$}

Now we focus attention on the thermodynamics, kinetics, and structure of the U-Zr configurations. We start with a random U-Zr configuration (also known as disordered configuration), which is allowed to naturally order with the given potential. Here we simulate the U-Zr alloy with iterative MC and MD simulations. The MC moves the ensemble to the lowest energy in an NVT ensemble. The same configuration is relaxed in MD to enable volumetric relaxation. The simulation begins with a $100 \mathrm{ps}$ MD simulation in an isothermal-isobaric (NPT) ensemble. The resulting atomistic configuration is then used in an MC simulation in an canonical (NVT) ensemble for 500 steps per atom. Then the atomistic configuration is exchanged between the NPT MD simulation and the NVT MC simulation until the MC simulation has less than a $5 \mathrm{meV}$ energy per atom change over the $500 \mathrm{MC}$ steps per atom. The simulation ends with a final NPT MD simulation, resulting in the final structure and properties that are reported. The evolution of the average energy per atom in the system during the MC simulations as the ordering progresses can be seen in Fig. 6 .

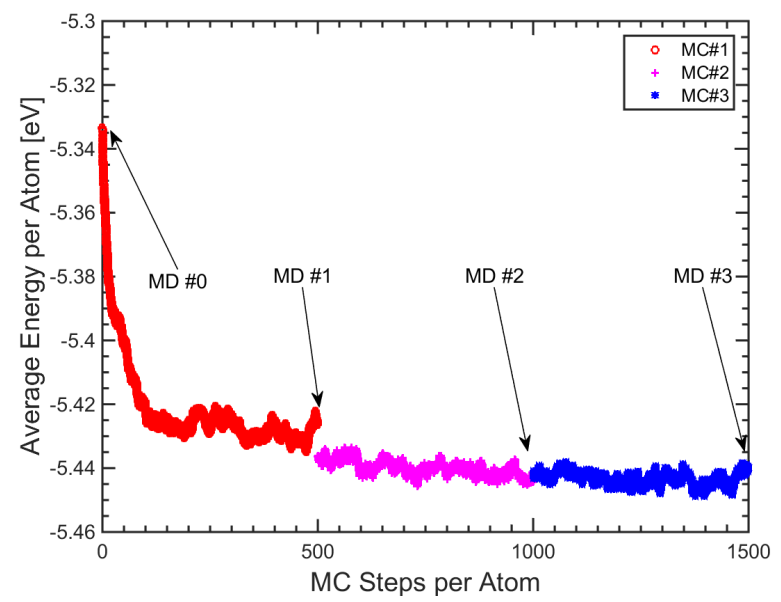

Figure 6: Average energy per atom of the U-20at.\%Zr system at $1100 \mathrm{~K}$ versus the number of MC steps per atom for each of the three MC simulations. The arrows show the configurations that are volumetrically relaxed using MD simulations.

The MD/MC simulations result in an ordered atomistic U-Zr configuration, which lowers the energy of the system. The average energy per atom is calculated at the end of the final MD simulation and the enthalpy of mixing, presented in Fig. 7 , shows a significant change from a disordered (random) U-Zr configuration to an ordered configuration. Thus, there is a thermodynamic driving force to order the U-Zr alloy, which could explain some of the separation and microstructural effects seen experimentally. 


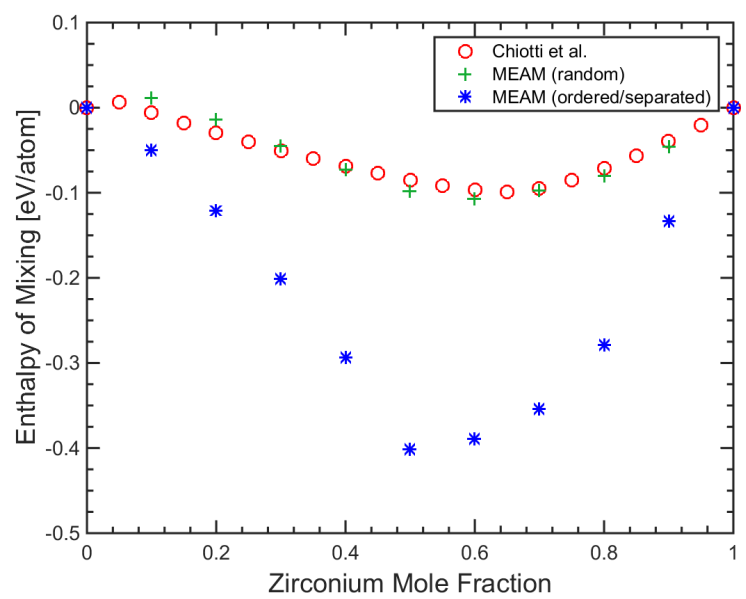

Figure 7: The enthalpy of mixing at $1100 \mathrm{~K}$ versus $\mathrm{Zr}$ mole fraction comparison for the random U-Zr configuration and the ordered/separated atomistic U-Zr configuration obtained using the MD/MC iterative simulations.

\subsection{Configurational Short Range Order}

In order to investigate the structure further, we compute the configurational SRO. The SRO of a few U-Zr alloys with respect to temperature gives an overview of the overall separation or ordering and how it is affected by temperature and concentration. The SRO parameter $(\sigma)$ considers only $1 \mathrm{NN}[43,45]$.

$$
\sigma=-\frac{P_{A A}-n_{A}}{1-n_{A}}
$$

$\mathrm{P}_{A A}$ is the fraction of the nearest neighbor sites of atom type $\mathrm{A}$ that is occupied by $\mathrm{A}$ type atoms (averaged over all $\mathrm{A}$ atoms). $\mathrm{n}_{A}$ is the atomic fraction of $\mathrm{A}$ type atoms in the entire system. The SRO allows a single number to represent a large amount of order/disorder phenomena. Complete atomic separation of an infinite system results in $\sigma=-1$, while an ordered system of opposite $1 \mathrm{NN}$ results in $\sigma$ $=1$.

In Fig. 8 it is seen that in the U-rich composition region the SRO parameter is negative and hence there is $\mathrm{U}$ and $\mathrm{Zr}$ separation. Then as the $\mathrm{Zr}$ concentration increases, the $\mathrm{SRO}$ also begins to increase, leading to the more ordered section around the intermediate composition region. The ordering around the intermediate composition region is due to the thermodynamic driving force to partial ordering seen in the $\delta-\mathrm{UZr}_{2}$ C32 phase. Lastly, the SRO begins to decrease due to the partially ordered sections beginning to separate from the Zr bcc matrix in the Zr-rich composition region.

As seen in Fig. 8 , the SRO of the bcc solid solutions tends to increase with temperature. This increase in SRO can be attributed to the changing morphology and the deviation away from alternating (111) planes of atoms, which causes a push of the SRO closer to zero. The liquid solutions tend to be more random, causing the SRO to approach a zero value. 


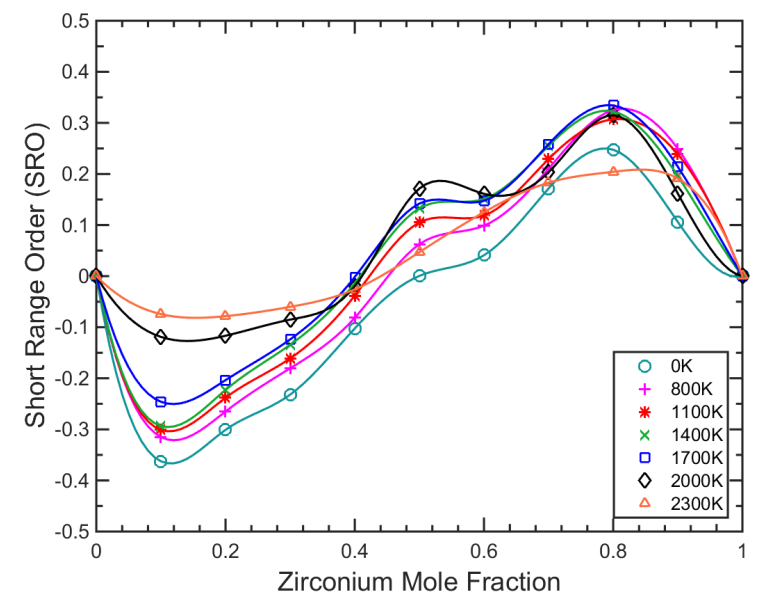

Figure 8: The SRO parameter of U-Zr alloy at $0 \mathrm{~K}, 1100 \mathrm{~K}, 1700 \mathrm{~K}, 2000 \mathrm{~K}$, and $2300 \mathrm{~K}$ versus mole fraction of zirconium.

\subsection{Ordering, Separation and Precipitate Morphology}

We can also study the ordering and separation morphology of the U-rich, intermediate, and Zr-rich U-Zr alloys. The ordering and precipitate morphology can be visualized as seen from the cross-sections of the atomistic configurations. These visualizations show the thermodynamic driving force toward certain separation or ordering behavior.

Atomistic configurations can be broken up into three regions: the U-rich region corresponding to the U$\mathrm{Zr}$ concentrations on the U-rich side of the $\delta$ - $\mathrm{UZr}_{2}$ phase (around 2 to 63 at.\% Zr), the intermediate region within the $\delta$ - $\mathrm{UZr}_{2}$ phase concentrations (around 64 to 78 at.\% Zr), and the Zr-rich region corresponding to the concentrations on the Zr-rich side of the $\delta-\mathrm{UZr}_{2}$ phase (around 78 to 91 at.\% $\mathrm{Zr}$ ).

Ordering analysis is conducted using four methods: the SRO parameter allowing for general ordering and separation trends, RDFs, BADFs, and visual analysis. Full and partial $\mathrm{g}(\mathrm{r})$ and $\mathrm{g}_{3}(\theta)$ distributions were calculated at various temperatures for the U-Zr alloys, offering more information on the structure and ordering of the U-Zr system. The partial BADFs consisted of six unique atomistic local configurations: U-U-U, U-Zr-U, U-U-Zr, Zr-Zr-Zr, Zr-U-Zr, and U-Zr-Zr. The BADFs were calculated using a $4.2 \AA \mathrm{R}_{m}$.

\subsubsection{Uranium-Rich Compositions}

The atomistic configurations (Fig. 9) of the U-rich U-Zr alloys show the spheroidization of the precipitates at lower temperatures versus the band-like lamellar precipitation at high temperatures, while Fig. 10 and Fig. 11 show the complimentary RDFs and BADFs respectively. The U-10at.\%Zr alloy is used to illustrate the typical U-rich precipitation phenomena. The atomistic configuration of the U-10at.\% Zr at 0K (Fig. 9a) illustrates alternating $\mathrm{U}$ and $\mathrm{Zr}$ (111) planes of atoms in a faceted spherical-like arrangement that has precipitated from the U-Zr alloy. The faceted precipitate was found to have an approximately $25 \AA$ size in the $<100>$ directions and $21 \AA$ size in the $<110>$ directions. This behavior shows the thermodynamic driving force toward the spherical precipitates observed experimentally [5, 7, 8, 46, 49] and 
shown in Fig. 1c. The precipitate has some preferred surfaces of separation on the (111), (221), and (110) surfaces. As the Zr concentration increases, the faceted separation continues at low temperatures, however the morphology changes from the alternating planes being surrounded by $\mathrm{U}$ atoms to the $\mathrm{U}$ atoms being surrounded by alternating planes.

As the temperature increases, the morphology of the (111) alternating $\mathrm{U}$ and $\mathrm{Zr}$ planes begin to change. The precipitate begins to form band-like arrangements. The atomistic configuration of U-10at.\% $\mathrm{Zr}$ at 1400K illustrates alternating $\mathrm{U}$ and $\mathrm{Zr}$ (111) planes of atoms in a band-like arrangement (Fig. 9c). The band-like precipitate was found to have an average thickness of about $12 \AA$ in the [001] direction. These (111) atom planes can also be seen in the Zr-Zr-Zr, U-Zr-U, and Zr-U-Zr g g $(\theta)$ (Fig. 11). The Zr-Zr-Zr shows a distinctive singular peak around $109^{\circ}$, indicating $1 \mathrm{NN}$ Zr atoms lining up in a particular direction, and the Zr-Zr g(r) (Fig. 10) shows that the Zr atoms are 1NN. Evidence of the 1NN Zr atoms lining up in a particular direction is also given by the $\mathrm{U}-\mathrm{Zr}-\mathrm{U}$ and $\mathrm{Zr}-\mathrm{U}-\mathrm{Zr}$ figures with their larger than average $90^{\circ}$ peaks. These bands are easily seen in the [100] direction; however, the surfaces of preferred separation are still (111) and (110). As the temperature increases even higher, the precipitation remains in the stable bcc band-like structure, but the separated uranium begins to change from bcc to a more liquid state. The band-like atomistic configuration shows the thermodynamic driving force to the lamellar [1 3 , and acicular [5, 7, 8, 46, 47, 50, 51] microstructures observed experimentally and shown in Fig. 1a and Fig. $1 \mathrm{~b}$

Above the melting point, there is an increase in atomistic motion, leading to an increase in atomic diffusion, that results in a random configuration of $\mathrm{U}$ and $\mathrm{Zr}$ atoms in the liquid structure (Fig. 9d). The transition into atomistic randomness is captured by the $g_{3}(\theta)$ in Fig. 11, where both the total and partial BADFs move to the characteristic shape of a binomial distribution with peaks around $55^{\circ}$ and $110^{\circ}$ with a trailing tail, indicating the liquid phase consists of a network of tetrahedrals.

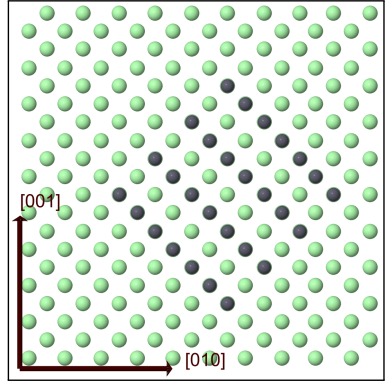

(a)

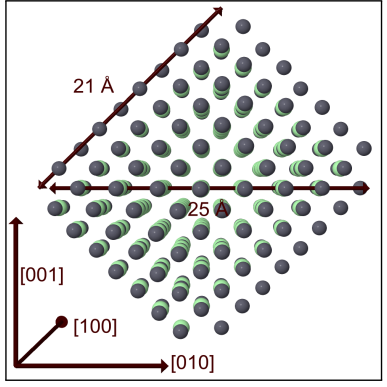

(b)

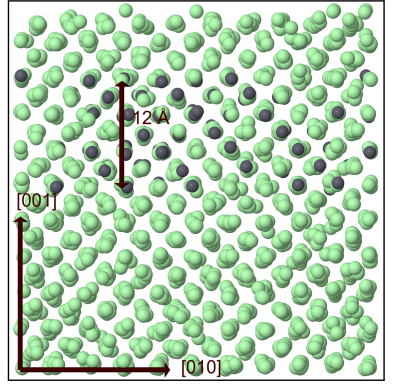

(c)

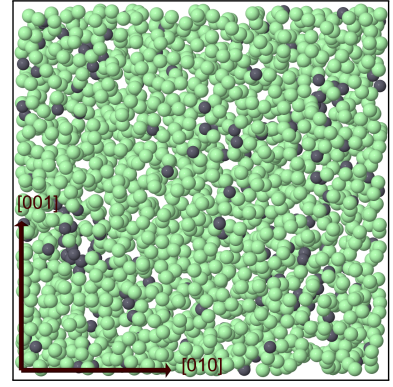

(d)

Figure 9: [100] Snapshots of equilibrium U(light-green)-10at.\%Zr(dark-grey) configurations at (a) 0K, (b) 0K precipitate, illustrating the alternating $\mathrm{U}$ and $\mathrm{Zr}$ (111) planes of atoms in a faceted spherical-like morphology, (c) 1400K illustrating the band-like separation of the alternating U and Zr (111) planes, and (d) 2000K illustrating the random liquid phase atomistic configuration. 

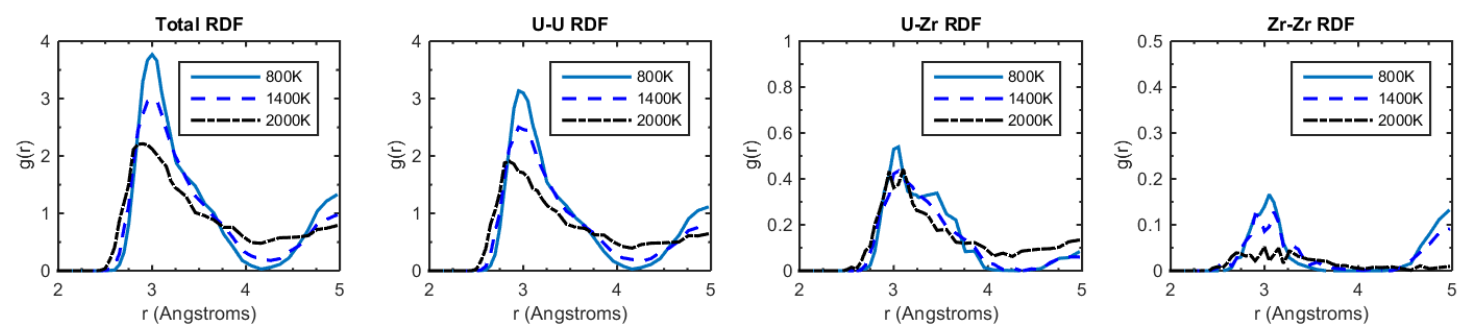

Figure 10: Full and partial RDFs of U-10at.\%Zr at 800K, 1400K, and 2000K.
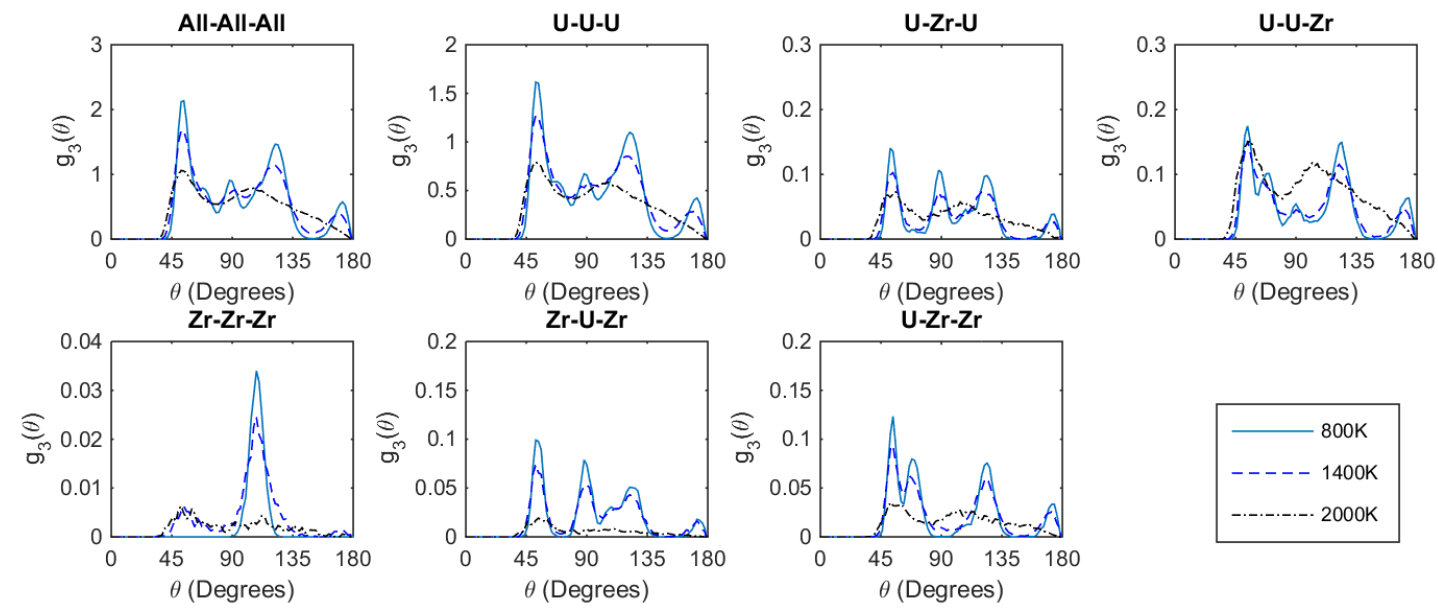

Figure 11: Full and partial BADFs of U-10at.\%Zr at 800K, 1400K, and 2000K.

The atomistic configurations of U-20at.\%Zr, U-30at.\%Zr, and U-40at.\%Zr at 1400K (Fig. 12 are used to illustrate the typical morphological transition as $\mathrm{Zr}$ concentration increases at temperature. As the $\mathrm{Zr}$ concentration increases, the alternating $\mathrm{U}$ and $\mathrm{Zr}$ (111) planes forming the band-like precipitates begin to broaden with U-20at.\%Zr having an average thickness of approximately $17 \AA$, and U-30at.\%Zr having an average thickness of approximately $22 \AA$. Then as the zirconium content increases to U-40at.\%Zr, the band-like separation transitions to a tubular morphology with an average diameter of about $17 \AA$. This tubular separation behavior, when taken as two-dimensional cross-sections, can be likened to the microstructure of the U-rich region approaching the compositions just outside of the intermediate region, which tends to have small discs and globules of separated U [8, 9]. 


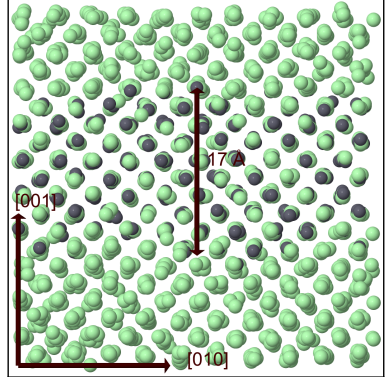

(a)

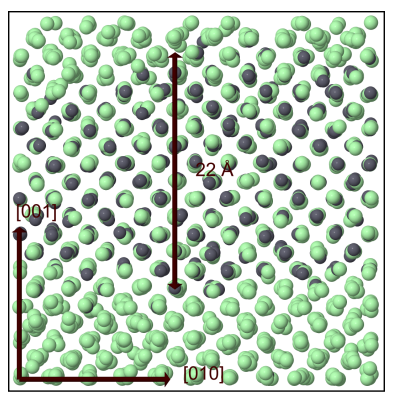

(b)

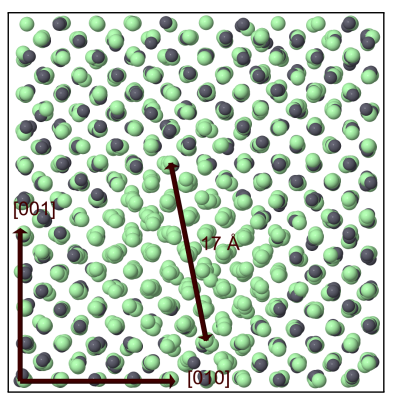

(c)

Figure 12: [100] Snapshots of 1400K equilibrium configurations of (a) U(light-green)-20at.\%Zr(dark-grey), (b) U-30at.\%Zr, and (c) U-40at.\%Zr. The atomistic configurations of U-20at.\%Zr and U-30at.\%Zr illustrate the broadening of the bandlike separation morphology consisting of alternating (111) U and Zr planes of atoms as Zr concentration increases, and U-40at.\%Zr illustrates the tubular separation morphology of pure U.

\subsubsection{Intermediate Compositions}

The atomistic configurations of the intermediate $\mathrm{U}-\mathrm{Zr}$ alloy within the $\delta$ - $\mathrm{UZr}_{2}$ phase concentration show more ordered atomic arrangement without precipitates (Fig. 13), while Fig. 14 and Fig. 15 show the complimentary RDFs and BADFs respectively. The U-70at.\%Zr alloy is used to illustrate the typical intermediate U-Zr alloy ordering phenomena. The atomistic configurations of U-70at.\%Zr from 0K-1400K show the bcc structure with a preference for short sections of $\mathrm{U}$ and $\mathrm{Zr}$ (111) alternating planes and an overall preference for alternate $1 \mathrm{NN}$ types as seen in the SRO parameter (Fig. 8) and the partial U-U $\mathrm{g}(\mathrm{r})$ (Fig. 14). The atomistic snapshots in Fig. 13 and the partial U-U g(r) depict that there is an ordering preference for $\mathrm{U}$ atoms to be $3 \mathrm{NN}$ and $4 \mathrm{NN}$. This overall preference for opposite type $1 \mathrm{NN}$ and $\mathrm{U} 3 \mathrm{NN}$ and $4 \mathrm{NN}$ is important when considering the transition from bcc to the $\delta-\mathrm{UZr}_{2}$ phase through the omega transformation mechanism. During the omega transformation mechanism, alternating (111) planes of the bcc phase collapse to form the $\mathrm{AlB}_{2}$ type hexagonal crystal structure, which has been documented by Basak et al. [52. In the partially ordered $\delta$ phase, the $\mathrm{Zr}$ atoms were found to occupy the $(0,0,0)$ position, and a random mixture of $\mathrm{U}$ and $\mathrm{Zr}$ atoms were found to occupy the $(2 / 3,1 / 3,1 / 2)$ and $(1 / 3,2 / 3,1 / 2)$ positions. The current atomistic configuration in the bcc structure allows for a more direct transition to the partially ordered $\delta$ phase. No atomistic precipitation is witnessed in this intermediate region, allowing the microstructure to result in equiaxed grains as observed experimentally and shown in Fig. 1d,

The RDFs (Fig. 14) and BADFs (Fig. 15) of the intermediate U-Zr system show the characteristic $55^{\circ}$ and $110^{\circ}$ peaks, indicating the liquid phase consists of a network of tetrahedrals. In addition, the partial RDFs and BADFs show the transition to atomistic randomness as temperature increases.

An ordered bcc phase with the $\mathrm{U}$ atoms surrounded by $\mathrm{Zr} 1 \mathrm{NN}$ and $2 \mathrm{NN}$, and $3 \mathrm{NN}$ being $\mathrm{U}$, can be reoriented with the bcc $P m \overline{3} \mathrm{~m}$ [111] direction equating to the $\mathrm{P} \overline{3} \mathrm{~m} 1$ [0001] of an equivalent trigonal 
unit cell (Fig. 16). Using this equivalent unit cell, the collapsing of the alternate bcc (111) planes can be easily visualized.

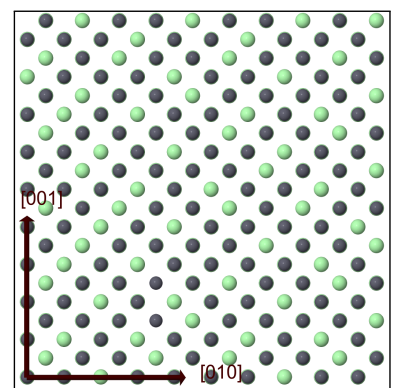

(a)

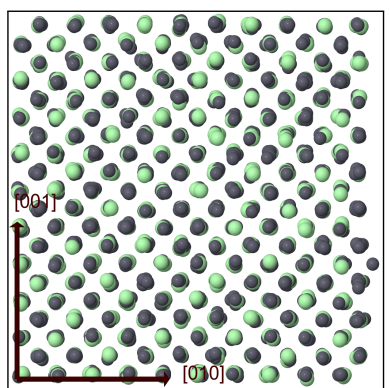

(b)

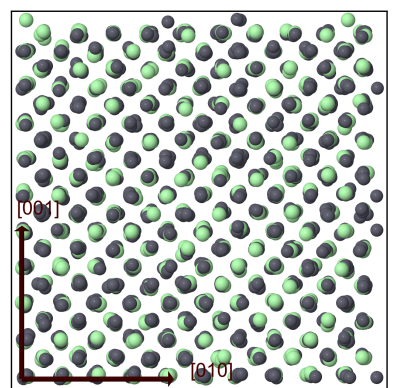

(c)

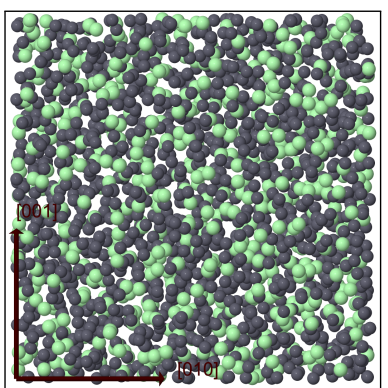

(d)

Figure 13: [100] Snapshots of equilibrium U(light-green)-70at.\%Zr(dark-grey) configurations at (a) 0K, (b) 800K, (c) 1400K, and (d) 2000K. The 0K-1400K snapshots illustrates the ordered atomistic configurations where there is a preference for $\mathrm{U}$ atoms to be $3 \mathrm{NN}$ or $4 \mathrm{NN}$, while $2000 \mathrm{~K}$ snapshot illustrates the transition to melting.
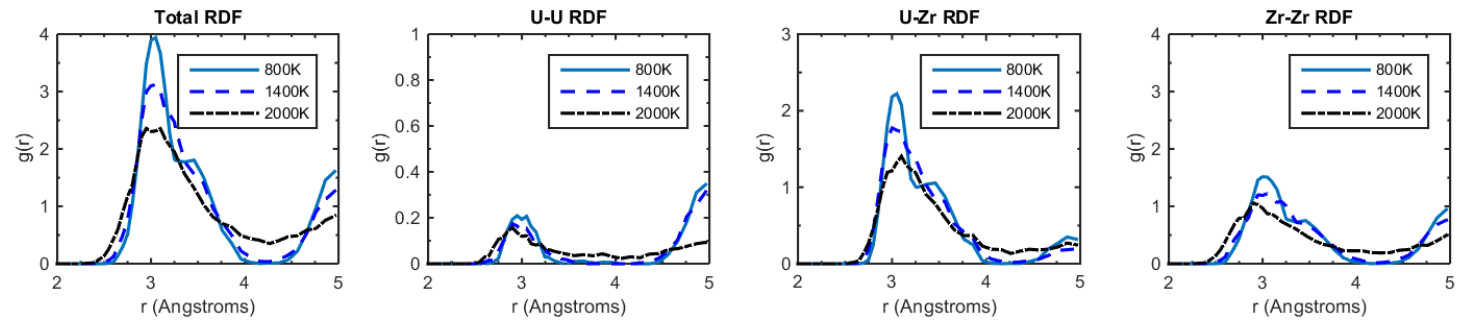

Figure 14: Full and partial RDFs of U-70at.\%Zr at $800 \mathrm{~K}, 1400 \mathrm{~K}$, and $2000 \mathrm{~K}$.
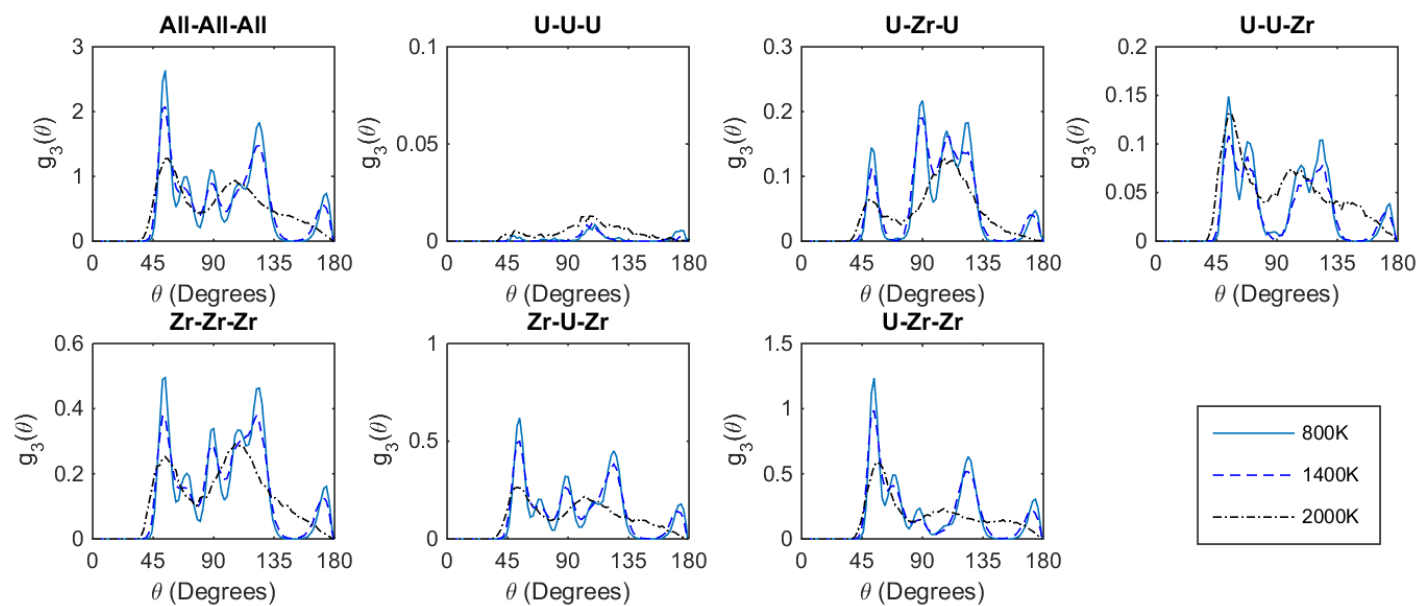

Figure 15: Full and partial BADFs of U-70at.\%Zr at 800K, 1400K, and 2000K. 


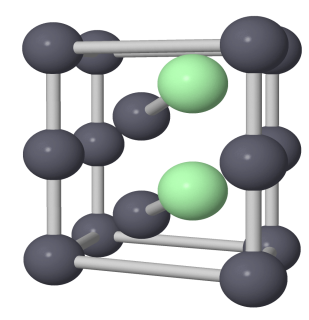

(a)

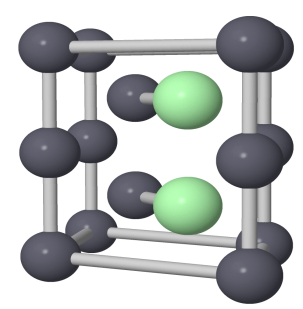

(b)

Figure 16: Snapshot of the (a) $\mathrm{P} \overline{3} \mathrm{~m} 1$ space group equivalent to a reoriented bcc structure with the $\mathrm{U}$ atoms (light-green) being $3 \mathrm{NN}$ to (b) the $\mathrm{P} 6 / \mathrm{mmm}$ space group of a partially ordered $\delta-\mathrm{UZr}_{2}$ phase.

\subsubsection{Zirconium-Rich Compositions}

The atomistic configurations of the Zr-rich U-Zr alloys (Fig. 17) show the precipitation of more bandlike behavior from 0K-2000K, while Fig. 18 and Fig. 19 show the complimentary RDFs and BADFs respectively. The U-90at.\%Zr alloy is used to illustrate the typical Zr-rich precipitation phenomena. The band-like separation is easily viewed from the [100] direction, and consists of regions of Zr atoms and regions of $\mathrm{U}$ and $\mathrm{Zr}$ atoms in differing arrangements where the $\mathrm{U}$ atoms are not $1 \mathrm{NN}$. The band-like structure has an average thickness of about $16 \AA$ at $800 \mathrm{~K}$ which becomes less defined as temperature increases, resulting in a chaotic band-like structure at $2300 \mathrm{~K}$. The preference of $\mathrm{U}$ atoms not being $1 \mathrm{NN}$ can also be seen in the U-U g(r) (Fig. 18) and the U-U-U, U-U-Zr, U-Zr-U, and Zr-Zr-Zr g g ( $\theta$ ) (Fig. 19). The U-U-U and the U-U-Zr $g_{3}(\theta)$ BADFs' lack of bond angles and the U-U g(r) figure's lack of radial information indicates that there are very few $\mathrm{U}-\mathrm{U} 1 \mathrm{NN}$ or $2 \mathrm{NN}$. However, from the $\mathrm{U}-\mathrm{Zr}-\mathrm{U} \mathrm{g}_{3}(\theta)$ figure, it can be seen that the U-Zr-U configuration has a tendency to line up in a single $1 \mathrm{NN}$ and a single $2 \mathrm{NN}$ direction from the $109^{\circ}$ and $122^{\circ}$ peaks. The $\mathrm{U}-\mathrm{Zr}-\mathrm{U} \mathrm{g}_{3}(\theta)$ figure and the atomistic configurations indicate that $\mathrm{U}$ atoms prefer to be $3 \mathrm{NN}$ and $4 \mathrm{NN}$. As explained in the Intermediate section (3.4.2), the $\mathrm{U}$ atoms' preference to not be $1 \mathrm{NN}$ but instead $3 \mathrm{NN}$ or $4 \mathrm{NN}$, makes for an easier transition to the partially ordered $\delta$ phase. The band-like precipitation behavior shows the thermodynamic driving force to the acicular, Widmanstätten, and martensitic needle microstructures observed experimentally [7, 8] and shown in Fig. 1e. 


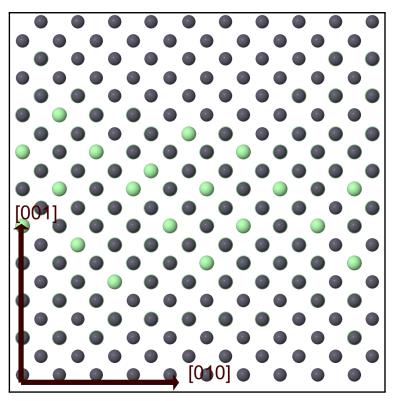

(a)

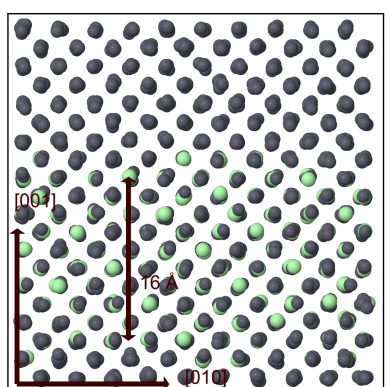

(b)

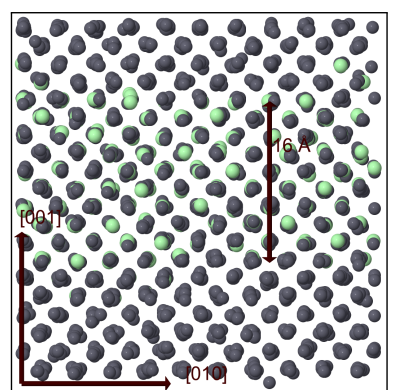

(c)

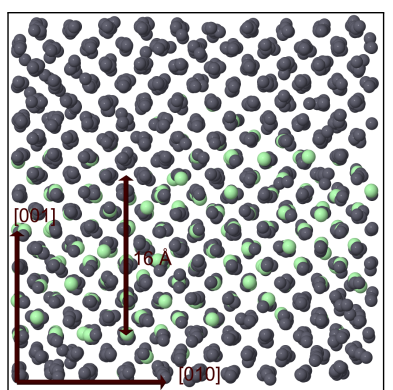

(d)

Figure 17: [100] Snapshots of equilibrium U(light-green)-90at.\%Zr(dark-grey) configurations at (a) 0K, (b) 800K, (c) 1400K, and (d) 2000K. The snapshots illustrate the band-like separation behavior of the regions where U atoms prefer to be $3 \mathrm{NN}$ or $4 \mathrm{NN}$ from the regions of pure $\mathrm{Zr}$.
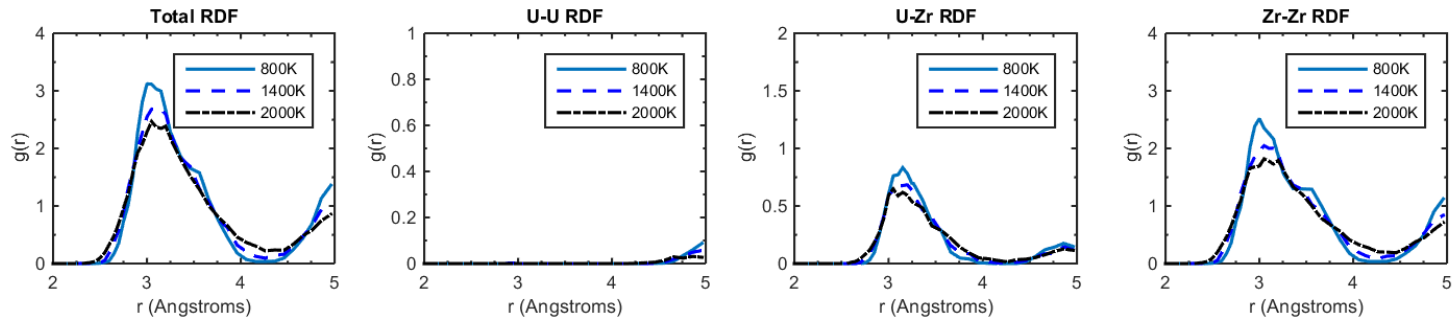

Figure 18: Full and partial RDFs of U-90at.\%Zr at 800K, 1400K, and 2000K.
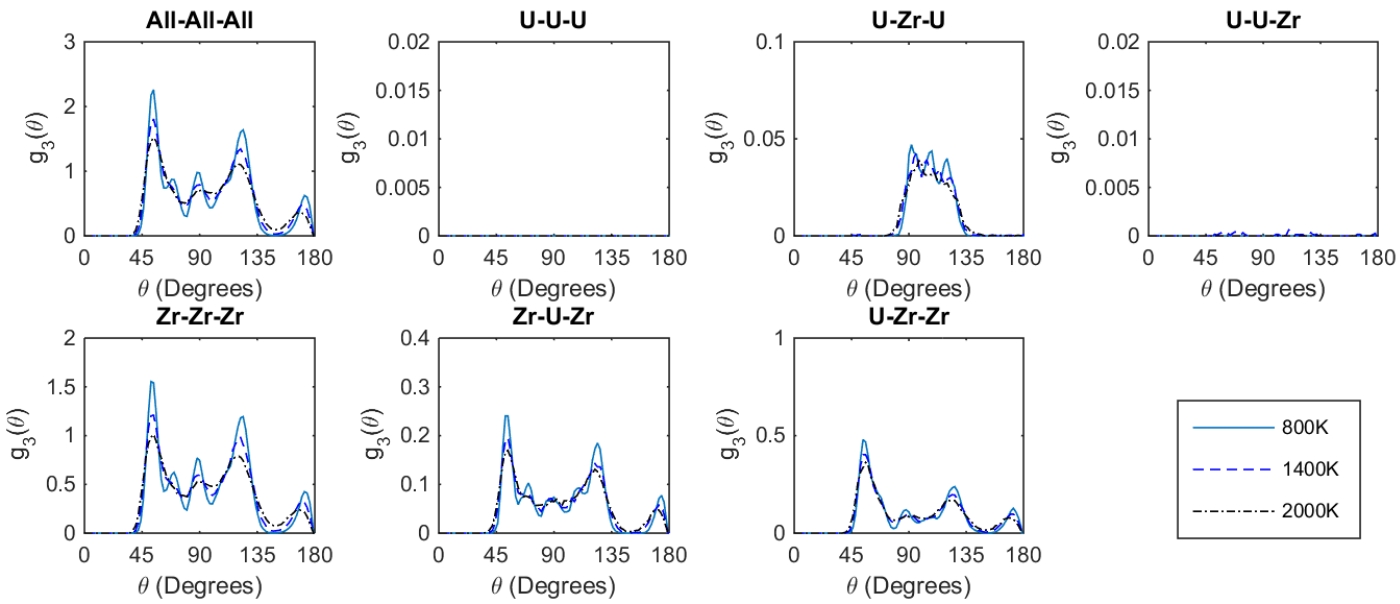

Figure 19: Full and partial BADFs of U-90at.\%Zr at 800K, 1400K, and 2000K.

\section{Conclusions}

Atomistic simulations of the thermodynamic ordering in a mixture are shown to be able to mimic and contribute to the deeper understanding of precipitation morphology and microstructure evolution 
in U-Zr alloys. In this paper we investigated the preferential ordering behavior of U-Zr alloys using an empirical potential and were able to relate the ordering behavior to observed microstructures. First, a comparison of $\gamma-\mathrm{U}$ and liquid-U structures is conducted and compared to previously published QMD and EAM results. The QMD and MEAM results show similar behaviors for both the bcc and liquid phases. The liquid-U phase was found to have a characterizable structure of a local network of tetrahedrals. Atomistic simulations of the U-rich alloys show a tendency towards separation resulting in precipitates whose shape depends on temperature. At lower temperatures the precipitates tend to form in more faceted and spherical arrangements, while at higher temperatures precipitates form in more of a band-like structure. Previous experimental research has shown this precipitate behavior change from spherical to lamellar microstructure as temperature increased, but until now this transition was not well understood. Intermediate alloys, around the $\delta$ - $\mathrm{UZr}_{2}$ phase concentration, show a preference to ordering where the $\mathrm{U}$ atoms are not $1 \mathrm{NN}$, but $3 \mathrm{NN}$ or $4 \mathrm{NN}$. This kind of ordering behavior allows for a more direct transition to the partially ordered $\delta-\mathrm{UZr}_{2}$ phase seen experimentally. Lastly, simulations of the Zr-rich alloys show a mix of ordering and separation behaviors. The Zr-rich systems show ordered sections where $\mathrm{U}$ atoms are $3 \mathrm{NN}$ or $4 \mathrm{NN}$, allowing more of a direct transformation to the partially ordered $\delta-\mathrm{UZr}_{2}$ phase, separated by bands from the regions of pure Zr. These bands mimic the acicular, Widmanstätten, and martensitic needle microstructures seen in the previous experimental research. These simulations are able to successfully replicate some of the fundamental thermo-physical and microstructural characteristics following fabrication and irradiation of the U-Zr metallic fuels.

\section{Acknowledgments}

Authors acknowledge support from the Idaho National Laboratory (INL), the Domestic Nuclear Detection Office (DNDO), the Department of Energy Nuclear Energy University Program (DOE-NEUP), and the National Science Foundation (NSF).

\section{References}

[1] C. Basak, G. J. Prasad, H. S. Kamath, N. Prabhu, An evaluation of the properties of as-cast U-rich U-Zr alloys, Journal of Alloys and Compounds 480 (2) (2009) 857-862. doi:10.1016/j.jallcom. 2009.02.077.

[2] J. McKeown, S. Irukuvarghula, S. Ahn, M. Wall, L. Hsiung, S. McDeavitt, P. Turchi, Coexistence of the $\alpha$ and $\delta$ phases in an as-cast uranium-rich U-Zr alloy, Journal of Nuclear Materials 436 (1) (2013) 100-104. doi:10.1016/j.jnucmat.2013.01.313.

[3] J. Kim, H. Song, H. Kim, K. Kim, C. Lee, R. S. Fielding, Development of a new casting method to 
fabricate U-Zr alloy containing minor actinides, Journal of Radioanalytical and Nuclear Chemistry 299 (1) (2014) 103-109. doi:10.1007/s10967-013-2767-5.

[4] J. Huber, P. Ansari, The superconductivity of bcc U-Zr alloys, Physica B+ C 135 (1) (1985) 441-444. doi:10.1016/0378-4363(85) 90527-3

[5] G. Lagerberg, Phase transformations in a uranium-zirconium alloy containing 2 weight per cent zirconium, Journal of Nuclear Materials 9 (3) (1963) 261-276. doi:10.1016/0022-3115(63)90144-2.

[6] S. Kaity, J. Banerjee, M. R. Nair, K. Ravi, S. Dash, T. R. G. Kutty, A. Kumar, R. P. Singh, Microstructural and thermophysical properties of $\mathrm{U}-6 \mathrm{wt} . \% \mathrm{Zr}$ alloy for fast reactor application, Journal of Nuclear Materials 427 (13) (2012) 1-11. doi:10.1016/j.jnucmat.2012.03.045

[7] A. A. Bauer, An evaluation of the properties and behavior of zirconium-uranium alloys, Report, Battelle Memorial Inst., Columbus, Ohio (1959).

[8] F. Rough, An evaluation of data on zirconium-uranium alloys, Report, Battelle Memorial Inst., Columbus, Ohio (1955).

[9] M. Akabori, A. Itoh, T. Ogawa, F. Kobayashi, Y. Suzuki, Stability and structure of the $\delta$ phase of the U-Zr alloys, Journal of Nuclear Materials 188 (0) (1992) 249-254. doi:10.1016/0022-3115(92) 90480-9.

[10] A. C. Bagchi, G. J. Prasad, K. B. Khan, R. P. Singh, A study on zirconium rich uraniumzirconium alloys, Transactions of the Indian Institute of Metals 67 (1) (2014) 123-130. doi: $10.1007 / \mathrm{s} 12666-013-0326-\mathrm{y}$.

[11] Thermo-calc software ab (2013).

[12] B. Sundman, B. Jansson, J.-O. Andersson, The thermo-calc databank system, Calphad 9 (2) (1985) 153-190. doi:10.1016/0364-5916(85)90021-5.

[13] H. Okamoto, U-Zr (uranium-zirconium), Journal of Phase Equilibria and Diffusion 28 (5) (2007) 499-500. doi:10.1007/s11669-007-9155-1.

[14] A. T. Dinsdale, SGTE data for pure elements, Calphad 15 (4) (1991) 317-425. doi:10.1016/ 0364-5916(91) 90030-N

[15] P.-Y. Chevalier, E. Fischer, B. Cheynet, Progress in the thermodynamic modelling of the O-U-Zr ternary system, Calphad 28 (1) (2004) 15-40. doi:10.1016/j.calphad.2004.03.005.

[16] B. Blumenthal, The transformation temperatures of high-purity uranium, Journal of Nuclear Materials 2 (1) (1960) 23-30. doi:10.1016/0022-3115(60)90020-9 
[17] H. L. Yakel, Review of x-ray diffraction studies in uranium alloys, Report CONF-740205-9, Oak Ridge National Lab., Tenn.(USA) (1973).

[18] F. A. Rough, A. A. Bauer, Constitution of uranium and thorium alloys, Battelle Memorial Institute, 1958.

[19] B. W. Howlett, The alloy system uranium-titanium-zirconium, Journal of Nuclear Materials 1 (3) (1959) 289-299. doi:10.1016/0022-3115(59)90025-X.

[20] A. Knapton, The crystal structure of TiU2, Acta Crystallographica 7 (5) (1954) 457-458. doi: 10.1107/S0365110X54001405.

[21] D. Peterson, D. Beernstein, The uranium-hafnium system, Transactions of American Society for Metals 52 (1960) 763-80.

[22] R. Rundle, A. Wilson, The structures of some metal compounds of uranium, Acta Crystallographica 2 (3) (1949) 148-150. doi:10.1107/S0365110X49000400

[23] E. Boyko, The structure of the $\delta$ phase in the uranium-zirconium system, Acta Crystallographica 10 (11) (1957) 712-713. doi:10.1107/S0365110X57002492.

[24] H. Okamoto, Si-U (silicon-uranium), Journal of Phase Equilibria and Diffusion 34 (2) (2013) 167168. doi:10.1007/s11669-012-0183-0.

[25] M. Akabori, T. Ogawa, A. Itoh, Y. Morii, The lattice stability and structure of $\delta$-UZr2 at elevated temperatures, Journal of Physics: Condensed Matter 7 (43) (1995) 8249.

[26] J. Silcock, Intermediate phase in the uranium-zirconium system-discussion, Transactions of the American Institute of Mining and Metallurgical Engineers 209 (1957) 521-521.

[27] A. Moore, B. Beeler, C. Deo, M. Baskes, M. Okuniewski, Atomistic modeling of high temperature uranium-zirconium alloy structure and thermodynamics, Journal of Nuclear Materials 467 (2015) 802-819. doi:10.1016/j.jnucmat.2015.10.016.

[28] A. Landa, P. Söderlind, P. E. A. Turchi, Density-functional study of the U-Zr system, Journal of Alloys and Compounds 478 (12) (2009) 103-110. doi:10.1016/j.jallcom.2008.12.052.

[29] G. Bozzolo, H. O. Mosca, A. M. Yacout, G. L. Hofman, Y. S. Kim, Surface properties, thermal expansion, and segregation in the U-Zr solid solution, Computational Materials Science 50 (2) (2010) 447-453. doi:10.1016/j.commatsci.2010.09.002.

[30] G. Bozzolo, H. O. Mosca, A. M. Yacout, G. L. Hofman, Atomistic modeling of the interaction of cladding elements (Fe, Ni, Cr) with UZr fuel, Journal of Nuclear Materials 414 (2) (2011) 101-108. doi:10.1016/j.jnucmat.2011.01.016 
[31] G. Bozzolo, G. L. Hofman, A. M. Yacout, H. O. Mosca, Lanthanides migration and immobilization in U-Zr nuclear fuels, Journal of Nuclear Materials 425 (13) (2012) 188-192. doi:10.1016/j.jnucmat. 2011.05 .013 .

[32] W. Xie, W. Xiong, C. A. Marianetti, D. Morgan, Correlation and relativistic effects in U metal and U-Zr alloy: Validation of ab initio approaches, Physical Review B 88 (2013) 235128. doi: 10.1103/PhysRevB.88.235128.

[33] W. Xiong, W. Xie, C. Shen, D. Morgan, Thermodynamic modeling of the U-Zr system a revisit, Journal of Nuclear Materials 443 (13) (2013) 331-341. doi:10.1016/j.jnucmat.2013.07.034.

[34] A. P. Moore, B. Beeler, M. Baskes, M. Okuniewski, C. S. Deo, Atomistic ordering in body centered cubic uranium-zirconium alloy, in: MRS Proceedings, Vol. 1514, Cambridge Univ Press, 2013, pp. 27-35. doi:10.1557/opl.2013.517.

[35] P. Chiotti, V. Akhachinskij, I. Ansara, M. Rand, The chemical thermodynamics of actinide elements and compounds, part 5, the actinide binary alloys, IAEA, Vienna, Report, STI/PUB/424/5 (1981).

[36] W. G. Hoover, Canonical dynamics: equilibrium phase-space distributions, Physical Review A 31 (1985) 1695-1697. doi:10.1103/PhysRevA.31.1695.

[37] S. Nosé, A molecular dynamics method for simulations in the canonical ensemble, Molecular physics 52 (2) (1984) 255-268. doi:10.1080/00268978400101201.

[38] N. Metropolis, A. W. Rosenbluth, M. N. Rosenbluth, A. H. Teller, E. Teller, Equation of state calculations by fast computing machines, The Journal of Chemical Physics 21 (6) (1953) 1087-1092. doi:10.1063/1.1699114

[39] G. Wang, M. A. Van Hove, P. N. Ross, M. I. Baskes, Monte Carlo simulations of segregation in Pt-Re catalyst nanoparticles, The Journal of Chemical Physics 121 (11) (2004) 5410-5422. doi: doi:10.1063/1.1781151.

[40] R. Q. Hood, L. Yang, J. A. Moriarty, Quantum molecular dynamics simulations of uranium at high pressure and temperature, Physical Review B 78 (2008) 024116. doi:10.1103/PhysRevB.78.024116.

[41] D. Smirnova, S. Starikov, V. Stegailov, Interatomic potential for uranium in a wide range of pressures and temperatures, Journal of Physics: Condensed Matter 24 (1) (2012) 015702.

[42] G. Kresse, J. Hafner, Ab initio molecular dynamics for liquid metals, Physical Review B 47 (1993) 558-561. doi:10.1103/PhysRevB.47.558.

[43] J. Cowley, X-ray measurement of order in single crystals of Cu3Au, Journal of Applied Physics 21 (1) (1950) 24-30. doi:10.1063/1.1699415. 
[44] F. Ducastelle, F. Ducastelle, Order and phase stability in alloys, North-Holland Amsterdam, 1991.

[45] C. S. Deo, D. J. Srolovitz, Atomic-scale three-dimensional kinetic Monte Carlo simulation of organometallic vapor-phase epitaxy of ordered films, Physical Review B 63 (2001) 165411. doi: 10.1103/PhysRevB.63.165411.

[46] H. Chiswik, A. Dwight, L. Lloyd, M. Nevitt, S. Zegler, Advances in the physical metallurgy of uranium and its alloys, Report A/CONF. 15/P/713, Argonne National Lab., Lemont, Ill. (1958).

[47] W. D. Wilkinson, Uranium Metallurgy: Uranium corrosion and alloys, Vol. 2, Interscience Publishers, 1962.

[48] C.-T. Lee, J.-H. Park, T.-K. Kim, U.-J. Lee, B.-S. Lee, D.-S. Sohn, Thermal stability of coextruded U-Zr/Zr-Nb alloys, Journal of Nuclear Materials 373 (13) (2008) 275-279. doi:10.1016/ j.jnucmat.2007.06.006

[49] C. B. Basak, Microstructural evaluation of U-rich U-Zr alloys under near-equilibrium condition, Journal of Nuclear Materials 416 (3) (2011) 280-287. doi:10.1016/j.jnucmat.2011.06.011.

[50] D. Summers-Smith, The constitution of uranium-zirconium alloys, Journal of the Institute of Metals 83 (1955) 277-282.

[51] M. Kasnan, The study of manufacturing and ingot characterization of U-Zr alloys, Applied Mechanics and Materials 376 (2013) 23-29. doi:10.4028/www.scientific.net/AMM.376.23

[52] C. B. Basak, N. Prabhu, M. Krishnan, On the formation mechanism of UZr2 phase, Intermetallics 18 (9) (2010) 1707-1712. doi:10.1016/j.intermet.2010.05.006 


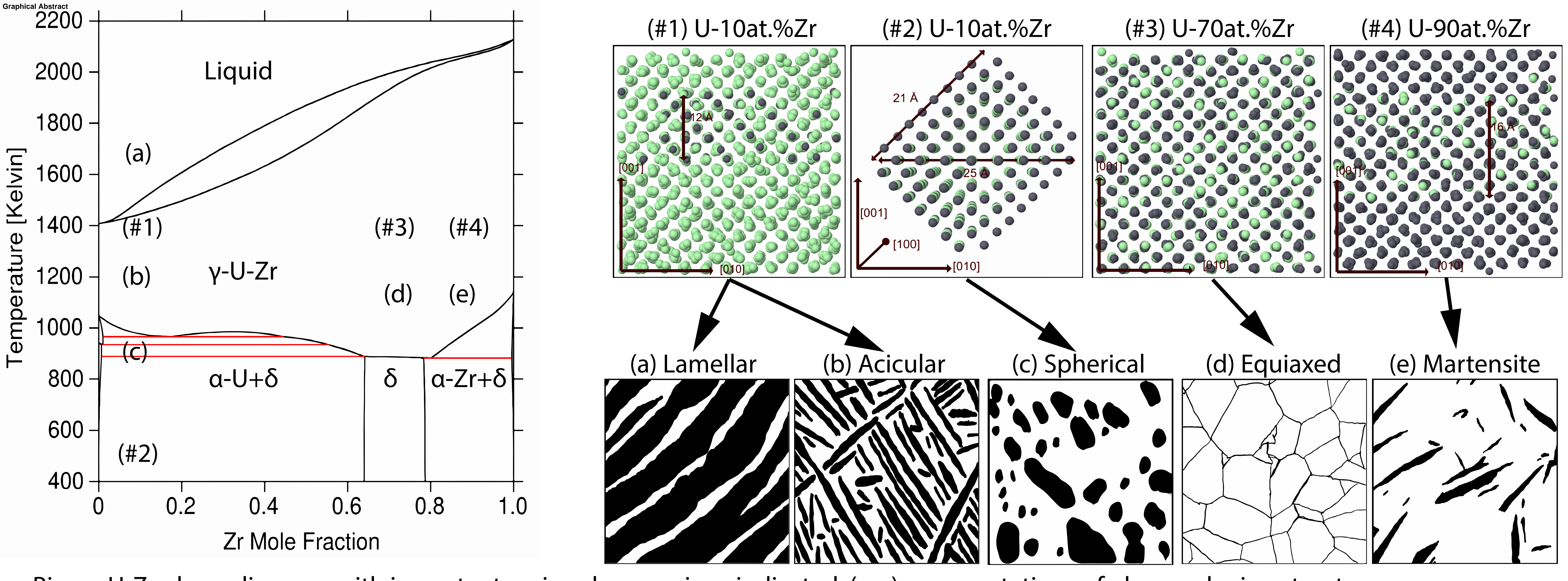

Binary U-Zr phase diagram, with important major phase regions indicated, (a-e) representations of observed microstructures if cooled with a given rate from a temperature range, and (\#1-4) atomistic configurations that lead to the observed microstructures. 